\title{
Input-driven bifurcations and information processing capacity in spintronics reservoirs
}

\author{
Nozomi Akashi $\odot,{ }^{1, *}$ Terufumi Yamaguchi, ${ }^{2}$ Sumito Tsunegi, ${ }^{3}$ Tomohiro Taniguchi $\odot,{ }^{3}$ Mitsuhiro Nishida, ${ }^{4}$ Ryo Sakurai, ${ }^{4}$ \\ Yasumichi Wakao $\odot,{ }^{4}$ and Kohei Nakajima ${ }^{1}$ \\ ${ }^{1}$ Graduate School of Information Science and Technology, The University of Tokyo, Bunkyo-ku, Tokyo, 113-8656, Japan \\ ${ }^{2}$ Yukawa Institute for Theoretical Physics, Kyoto University, Kyoto, 606-8502, Japan \\ ${ }^{3}$ National Institute of Advanced Industrial Science and Technology (AIST), Spintronics Research Center, Tsukuba, Ibaraki, 305-8568, Japan \\ ${ }^{4}$ Bridgestone Corporation, Tokyo, 104-8340, Japan
}

(Received 30 June 2020; revised 8 October 2020; accepted 6 November 2020; published 1 December 2020)

\begin{abstract}
Spintronics devices generate diverse nonlinear dynamics and have been studied as promising candidates for physical reservoir computing systems. However, the dynamic properties of spintronics reservoirs driven by input streams are largely yet to be uncovered. This study reveals that two types of bifurcation, from order to chaos and from chaos to order, can be induced by increasing the strength of input signals to the spintronics reservoir, and the information processing capacity of the reservoir changes drastically according to these bifurcations. The significant contributions of input-induced diversity in magnetization dynamics are demonstrated through numerical experiments, which include a real-world sensor emulation task. Our results suggest that modulating input settings can generate a diverse repertoire of magnetization dynamics without tuning the physical platform itself, providing valuable insights into neuromorphic applications.
\end{abstract}

DOI: 10.1103/PhysRevResearch.2.043303

\section{INTRODUCTION}

Physical reservoir computing (PRC) is a method that originated from schemes of recurrent neural network training $[1,2]$, and it exploits physical dynamics as computational resources [3]. Target substrates include a range of physics, from mechanical systems [4,5] to photonics [6-9] and quantum systems [10-12]. Among physical substrates, spintronics devices known as spin-torque oscillators (STOs) have recently gained attention due to their high-speed time scale, minute size, and high level of energy efficiency [13-19]. Spintronics devices are also known to be robust against soft errors under radiation exposure [20], making them good candidates for information processing in extreme environments, such as outer space and disaster zones, where conventional computers usually break down. The STO dynamics are based on limit cycles and include complex phenomena, such as bifurcations and synchronizations [21-30]. It was revealed recently that spintronics devices exhibit chaos when driven by a sinusoidal wave or feedback current fed into their dynamics [31-33].

In this paper, we aim to clarify the rich repertoire of STO dynamics as input-driven bifurcations and reveal their relevance to system information processing capability, which has been largely uncovered to date. A spintronics reservoir is represented as a nonlinear dynamical system injecting input signals when exploited as an information processing resource of PRC. We will show that two bifurcations, which are from

\footnotetext{
*akashi@isi.imi.i.u-tokyo.ac.jp
}

Published by the American Physical Society under the terms of the Creative Commons Attribution 4.0 International license. Further distribution of this work must maintain attribution to the author(s) and the published article's title, journal citation, and DOI. order to chaos and from chaos to order, can be induced by changing input conditions, such as input current magnitudes and switching intervals. The internal parameters of PRC, unlike input parameters, are difficult to change after fixing the device configuration, which contrasts with traditional neural networks. Therefore it would be beneficial to have diverse dynamics repertories generated only by input configuration. Furthermore, we will also show that the information processing capability of the spintronics reservoir changes drastically according to these bifurcations. We analyze these bifurcations numerically using the Lyapunov exponent and echo state property (ESP) [2,34]. The dynamics possess (linear) memory capacity (MC) [35] and information processing capacity (IPC) [36], which provide comprehensive criteria for analyzing linear and nonlinear MCs. As a result, we reveal that the MC and IPC properties change completely depending on bifurcations, and there are singular phenomena of $\mathrm{MC}$ at certain input intervals that are multiples of the original oscillation period of the STO dynamics. Accordingly, we demonstrate that performance of machine-learning tasks, which are emulations of nonlinear dynamical systems called the nonlinear autoregressive moving average (NARMA2) [37] when referred to as virtual tasks and called the length emulation of pneumatic artificial muscle (PAM) [38] when referred to as real-world tasks of edge computing, can be improved significantly only by tuning input parameters.

\section{SYSTEM DESCRIPTION}

Our STO model is shown in Fig. 1(a). The unit vectors point in the magnetization directions in the free and reference layers and are denoted as $\mathbf{m}$ and $\mathbf{p}$, respectively. The magnetization in the free layer autonomously oscillates and 


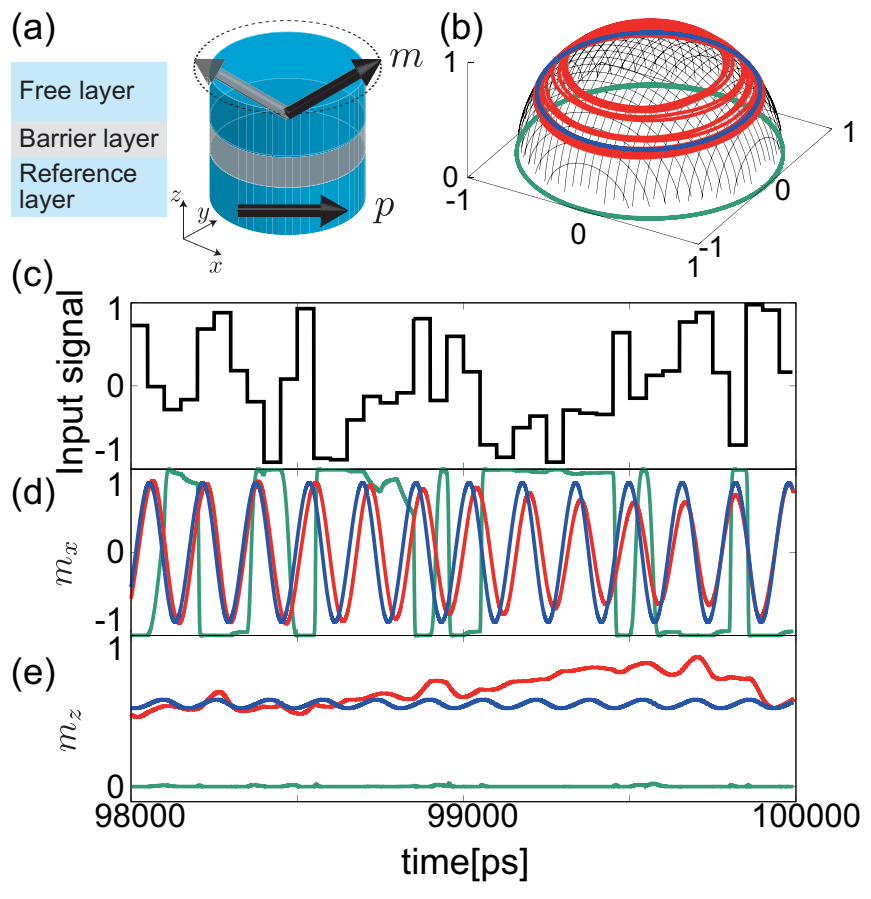

FIG. 1. Typical STO dynamics driven by input stream. (a) A schematic view of the system. (b) Typical dynamics of the magnetization $\mathbf{m}$ in a steady state in Cartesian coordinate space. The input interval is $\tau=50 \mathrm{ps}$, and the input magnitudes are $A=0.0,5.0$, and $500 \mathrm{~mA}$ (blue, red, and green, respectively). (c) Time series of input signal $j_{\text {in }}(t)$ injected to the STO in (b). (d) and (e) Time series with $x$ and $z$ components of the magnetization in the free layer, respectively. The color correspondence is the same as in (b).

is followed by the Landau-Lifshitz-Gilbert (LLG) equation, given by

$$
\begin{gathered}
\frac{d \mathbf{m}}{d t}=-\gamma \mathbf{m} \times \mathbf{H}-\gamma H_{s}(t) \mathbf{m} \times(\mathbf{p} \times \mathbf{m})+\alpha \mathbf{m} \times \frac{d \mathbf{m}}{d t}, \\
H_{s}(t)=\frac{\hbar \eta j(t)}{2 e(1+\lambda \mathbf{m} \cdot \mathbf{p}) M V},
\end{gathered}
$$

where $\gamma$ and $\alpha$ are the gyromagnetic ratio and the Gilbert damping constant, respectively. The magnetic field $\mathbf{H}=$ $\left[H_{\text {appl }}+\left(H_{\mathrm{K}}-4 \pi M\right) m_{z}\right] \mathbf{e}_{z}$ consists of an applied field $H_{\text {appl }}$, interfacial magnetic anisotropy field $H_{\mathrm{K}}$, and demagnetization field $-4 \pi M . M$ and $V$ are the saturation magnetization and the volume of the free layer, respectively. The spin-transfer torque strength $H_{s}$ is characterized by the spin polarization $\eta$ and spin-transfer torque asymmetry $\lambda$. The magnetization $\mathbf{p}$ in the reference layer is fixed to positive $x$. The current $j(t)$ added to the time-variant input signal $j_{\text {in }}(t)$ is given by $j(t)=$ $j_{\mathrm{dc}}+A j_{\mathrm{in}}(t)$, where $A$ is the input magnitude, $j_{\mathrm{dc}}=2.5 \mathrm{~mA}$ is a constant, and the input signal is $j_{\text {in }}(t)=u_{n}(n=\max \{n \in$ $\mathbb{Z} \mid n<t / \tau\}$ ), where $u_{n}$ is an independent and identically distributed (i.i.d.) random variable whose distribution function is a uniform distribution with an interval of $[-1,1]$ and $\tau>0$ as the input interval. This type of square input is common not only in spintronics reservoir computing [13-17] but also in other physical reservoirs generally $[6,9-11,39]$. Particularly,
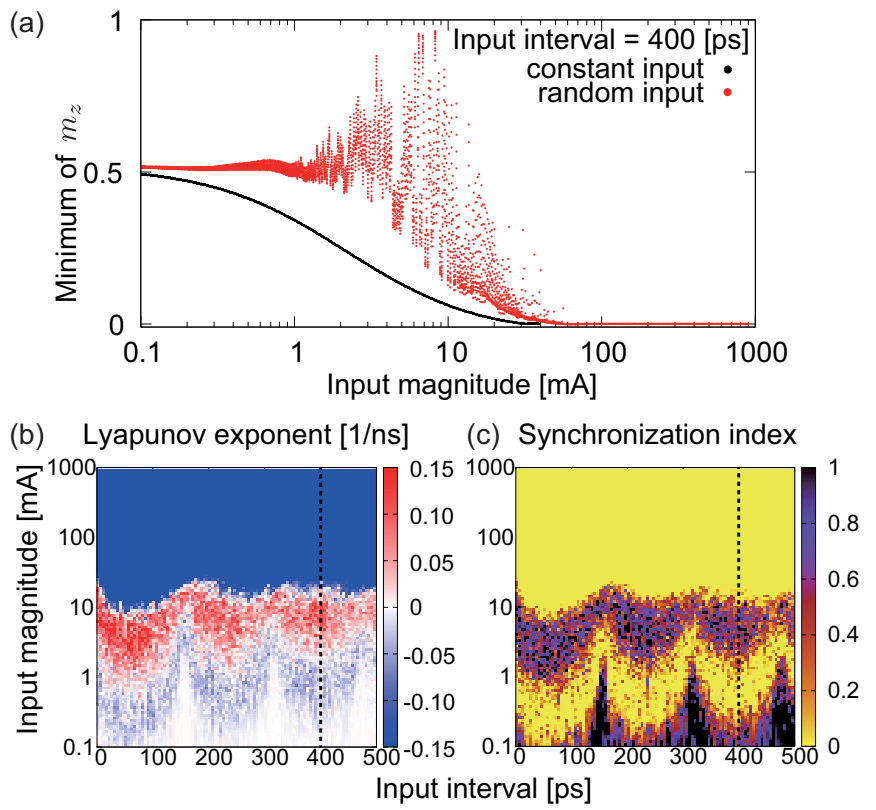

FIG. 2. Input-driven bifurcations and an echo state property. (a) Bifurcation diagram of the STO with input interval $\tau=400 \mathrm{ps}$. Local minimums of the $z$ component are plotted. The input signal is constant as $j=j_{\mathrm{dc}}+A$ for the black points and random as $j(t)=$ $j_{\mathrm{dc}}+A j_{\text {in }}(t)$ for the red points. (b) Color map of the conditional Lyapunov exponent. (c) Color map of the synchronization indices. The black dotted lines in (b) and (c) represent input conditions in (a).

the $\mathrm{MC}$ of spintronics reservoirs injected with binary square input signals has been studied theoretically $[14,19]$ and experimentally $[15,16]$. In this paper, all internal STO parameters are fixed, and we survey only external parameters of the input signal, which has input magnitude $A$ and input interval $\tau$. STO parameters are set to reflect a real-world setting and are derived from experiment [23] together with a theoretical analysis [40] (see Appendix A).

We numerically solve the LLG equation using the fourth Runge-Kutta method. Orbits of the LLG equation with three input conditions and the input signal are shown in Figs. 1(b)1(e). When the input is switched off with $A=0 \mathrm{~mA}$, the magnetization dynamics are periodic. When the input magnitude is $A=5.0 \mathrm{~mA}$, the dynamics fluctuates from the limit cycle of $A=0 \mathrm{~mA}$. When the input magnitude is extremely strong, $A=500 \mathrm{~mA}$, the magnetization switches between parallel and antiparallel alignments of the magnetizations in the free and reference layers [24,25]. $(x, y, z)=(-1,0,0)$ and $(1,0,0)$ are fixed points corresponding to when the sign of current is positive or negative, respectively. Hence the orbit rapidly switches between the two fixed points.

\section{BIFURCATIONS}

Here, we investigate input-driven bifurcations of the STO. The typical bifurcation diagram along the input magnitude is shown in Fig. 2(a). Local minimums of the $z$ component after transient time are plotted. The input signal is constant for the black points as $j=j_{\mathrm{dc}}+A$, whereas it is random for the red points as $j(t)=j_{\mathrm{dc}}+A j_{\text {in }}(t)$. In the constant input 
signal, the dynamics are always ordered. By contrast, given a random input signal, the dynamics become chaotic and show a pointlike spread once the input magnitude exceeds $1 \mathrm{~mA}$. Furthermore, when the input magnitude exceeds $30 \mathrm{~mA}$, the dynamics become orderly again and return to a line. This can be understood as two existing bifurcations: order to chaos and chaos to order.

To investigate these bifurcations quantitatively, we calculated the Lyapunov exponent, the criterion of initial state sensitivity of dynamics. A positive Lyapunov exponent is typically a feature of chaos. It should be noted that this system is nonautonomous; due to the time variant of the input signal, we calculated conditional Lyapunov exponents [41] that are restricted to the expanding rate of the magnetization $\mathbf{m}$ (see Appendix B). Figure 2(b) shows the color map of the largest conditional Lyapunov exponents for the STO. The red regions correspond to positive Lyapunov exponents, confirming chaos in certain input conditions. In addition, bifurcation points show periodic structures along the axis of the input interval. The input intervals $\tau=160,320$, and $480 \mathrm{ps}$, in which the chaos regions are shrunk, are multiples of the period $T=160$ ps of the limit cycle oscillation in the absence of the random input. If the input interval is $n T$, where $n$ is a positive integer, it is hard to induce chaos, but if the input interval is $n T-T / 2$, chaos can be induced with a smaller input magnitude. In white regions with zero Lyapunov exponents, the dynamics are pure limit cycle, and the input signal is never reflected.

At this point, we investigated an important reservoir property: ESP [2,34], which is defined so the current state of a reservoir is a function of the past input series. The ESP is a similar concept to generalized synchronization between an input signal and a system response, and it is confirmed by the occurrence of a common-signal synchronization [42-44]. We defined the following quantity, the synchronization index, to numerically confirm the ESP of STOs [43]:

$$
\mathcal{F}(R, S)=\frac{1}{S} \int_{R}^{R+S} \mathrm{~d}\left(\mathbf{m}_{1}(t), \mathbf{m}_{2}(t)\right) d t,
$$

where $R, S \gg 0$ are transient time and evaluation time, respectively, $\mathbf{m}_{1}(t), \mathbf{m}_{2}(t)$ are magnetizations with common input signals and different initial conditions, respectively, and $\mathrm{d}(\cdot, \cdot)$ is the distance for the magnetizations. If $R$ and $S$ are sufficiently large, $\mathcal{F}(R, S)$ are independent of $R$ and $S$. If the STO has common-signal synchronization, $\mathcal{F}(R, S)$ approximately equals zero, and the STO has the ESP. Figure 2(c) shows the color map of the synchronization index (parameters of the numerical analyses are shown in Appendix C). We confirmed that the synchronization index equals zero when the conditional Lyapunov exponent in Fig. 2(b) is negative. The result that the negative conditional Lyapunov exponent is the condition of the common-signal synchronization was theoretically proven by Refs. $[42,45]$.

\section{INFORMATION PROCESSING CAPABILITY}

To evaluate the system's information processing capability, we introduce two measures: MC [35] and IPC [36]. We define $X=\left\{\mathbf{x}_{n}\right\}$ as the output series of the system, where input series $\left\{u_{n}\right\}$ is injected. The capacity for a target function series $\left\{y_{n}\right\}=\left\{y\left(u_{n}, u_{n-1}, \ldots\right)\right\}$ is defined by the following equation:

$$
\begin{aligned}
C[X, y] & =1-\frac{\min _{\hat{y}_{n}} \operatorname{MSE}\left[y_{n}, \hat{y}_{n}\right]}{\left\langle y_{n}^{2}\right\rangle} \\
& =\frac{\left\langle y_{n} \mathbf{x}_{n}\right\rangle^{\mathrm{T}}\left\langle\mathbf{x}_{n} \mathbf{x}_{n}^{\mathrm{T}}\right\rangle^{-1}\left\langle y_{n} \mathbf{x}_{n}\right\rangle}{\left\langle y_{n}^{2}\right\rangle},
\end{aligned}
$$

where prediction value $\hat{y}_{n}$ is linearly transformed from output value $\mathbf{x}_{n}, \operatorname{MSE}\left[y_{n}, \hat{y}_{n}\right]$ is the mean-square error between $\left\{y_{n}\right\}$ and $\left\{\hat{y}_{n}\right\}$ through $n$, and $\left\langle y_{n}^{2}\right\rangle$ denotes the average of $y_{n}^{2}$ through $n .0 \leqslant C[X, y] \leqslant 1$ always holds [35]. When $C[X, y]=0$, the system never reconstructs target $y$, but when $C[X, y]=1$, the system can reconstruct target $y$ completely. If the input series is an i.i.d. random sequence with uniform distribution, $-1 \leqslant$ $u_{n} \leqslant 1, y\left(u_{n}, u_{n-1}, \ldots\right)$ can be decomposed into the following orthogonal polynomials: $\left\{y^{l}\left(u_{n}, u_{n-1}, \ldots\right)\right\}_{l=0,1, \ldots}$, which is

$$
y^{l}\left(u_{n}, u_{n-1}, \ldots\right)=\prod_{d=1} \mathcal{P}_{k_{l, d}}\left(u_{n-d}\right),
$$

where $\mathcal{P}_{k}(u)$ is the Legendre polynomial with degree $k$ and $k_{l, d} \geqslant 0$ is a series of degrees for $y^{l}$. We restrict the delay of input series $u_{n}, \ldots, u_{n-D}$ to $D$ and set $\mathcal{S}(D, K)$ of orthogonal basis function $y^{l}$ to total degree $K=\sum_{d=0}^{D} k_{l, d}$. Here, IPC and MC with delay $D$ and total degree less than or equal to $K$ are defined as follows:

$$
\begin{gathered}
\operatorname{IPC}[X, D, K]=\sum_{k=1}^{K} \sum_{d=0}^{D} \sum_{y^{l} \in \mathcal{S}(d, k)} C\left[X, y^{l}\right], \\
\operatorname{MC}[X, D]=\operatorname{IPC}[X, D, 1]=\sum_{d=0}^{D} \sum_{y^{l} \in \mathcal{S}(d, 1)} C\left[X, y^{l}\right] .
\end{gathered}
$$

The IPC is the sum of capacities for all orthogonal basis functions, and MC is restricted to linear basis functions. Particularly, the memory function is defined as $m(d)=$ $C\left[X, u_{n-d}\right]$, which is a decomposition of MC through delay $d$. For a number $N$ of the linear independent output of system $X$, the following two important equations hold [36]:

$$
\lim _{D \rightarrow \infty} \operatorname{MC}[X, D] \leqslant \lim _{D, K \rightarrow \infty} \operatorname{IPC}[X, D, K] \leqslant N
$$

and, if $X$ has ESP,

$$
\lim _{D, K \rightarrow \infty} \operatorname{IPC}[X, D, K]=N .
$$

We present the results of $\mathrm{MC}[X, 999]$ in Fig. 3 (parameters of the numerical analyses are shown in Appendix D). Here, the output series $\mathbf{m}_{n}$ is defined as the magnetization $\mathbf{m}_{n}=$ $\mathbf{m}((n+1) \tau)$ at the time of changing the next input signal. The color map of the MC is shown in Fig. 3(a). We present two interesting findings related to the bifurcations and the MC. First, the MC properties are completely different before and after bifurcations. Figures 3(b) and 3(c) show the MC graphs with input intervals $\tau=80$ and $\tau=160 \mathrm{ps}$, respectively, separated by delay components. Given an input interval equal to $\tau=$ $80=n T-T / 2$, where $n$ is a positive integer and $T=160 \mathrm{ps}$ is the oscillation period of the STO without random inputs, there are two peaks before and after chaos regions. This result is in accordance with a previous study [46] that found that MC improves at the edge of chaos. In an ESP region with a smaller 
(a)
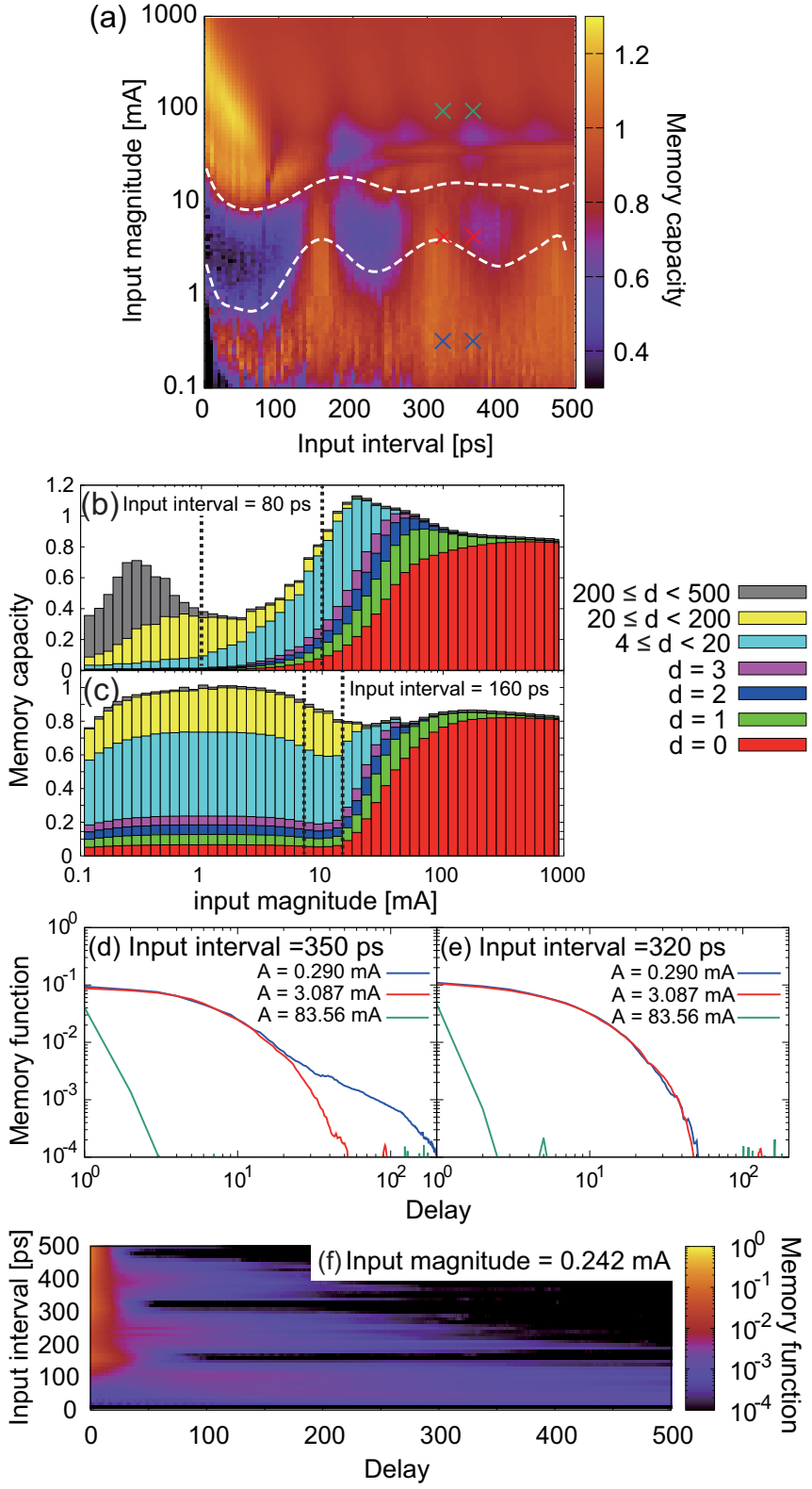

FIG. 3. Analyses of the memory capacity (MC). (a) Color map of the MC. White lines represent bifurcation points. Cross marks represent input conditions in (d) and (e). (b) and (c) MC properties with input interval (b) $\tau=80 \mathrm{ps}$ and (c) $\tau=160 \mathrm{ps}$. Red, green, blue, purple, light blue, yellow, and gray correspond to delay $d=0$, 1, 2, 3, 4-19, 20-199, and 200-499, respectively. Black dotted lines represent bifurcation points. (d) and (e) Memory functions $m(d)$ with an input interval of 350 and $320 \mathrm{ps}$, respectively. The green, red, and blue lines represent an input magnitude of $0.29 \mathrm{~mA}$ before the chaos region, an input magnitude of $3.087 \mathrm{~mA}$ in the chaos region, and an input magnitude of $83.56 \mathrm{~mA}$ after the chaos region, respectively. (f) Color map of the memory function $m(d)$ with input magnitude $A=0.242 \mathrm{~mA}$. The color represents capacity of the corresponding delay $d$ and input interval $\tau$.

input magnitude, capacities of extremely long delays greater than or equal to 200 and less than 500 are dominant. When the input magnitude increases and the chaos region is approached, the dynamics no longer have ESP, and the MC decreases.
The extremely long delay components from 200 to 499 are lost, making delay components from 20 to 199 predominant. Comparing an ESP region with a smaller input magnitude and chaos region in Fig. 3(d), we find that components up to delay 20 coincide and it loses extremely long delay components from delay 20. When the input strength is increased further and the ESP region is approached again after the bifurcation, the MC improves once more.

The second finding is that the $\mathrm{MC}$ decline in chaos regions does not occur when input interval $\tau=160,320,480=n T$ ps. Memory functions at input interval $\tau=320 \mathrm{ps}$ are shown in Fig. 3(e). Memory functions of ordered dynamics with a small input magnitude and chaos coincide in all delays. For input interval $\tau=160=n T$ ps, there are no peaks before or after the chaos regions. Given an ESP region with a small input magnitude, memory functions with extremely long delays vanish. In the ESP region with a large input magnitude, MC does not peak around the edge of bifurcation, and there is no decrease of MC in the chaos regions; therefore MC always has a high value in the input magnitude. Figure 3(f) shows memory functions for the input interval as a color map, confirming that the MC tail vanishes sharply at input intervals $\tau=n T$.

It is important to note that in recent studies of randomly coupled recurrent neural networks, the input-driven bifurcation and its relationship with MC were theoretically investigated in both continuous-time [47] and discrete-time [48] settings. These studies suggest that randomly coupled networks with sigmoid activation functions driven by random input streams only exhibit a bifurcation from chaos to order when the input magnitude increases and network MCs are modulated accordingly. Interestingly, our results have shown that spintronics reservoirs exhibit completely different bifurcation structures and MC behaviors compared with conventional neural networks, which have higher diversity in the dynamics.

Next, we show the results of the IPC. Figure 4(a) shows the color map of the IPC $[X, 4,29]$ for input conditions. IPC $=$ $N=3$ is not held in the ESP regions, as in Eq. (9). The reason for this is the restriction of the target function that is used for the IPC calculation. In the ESP regions where the input magnitude is smaller, there are long delay components with a delay from 20 to 500, but here, only target functions up to $D=4$ are calculated. In addition, in ESP regions where the input magnitude is larger, the IPC is close to 2.0. This is because not all degrees are calculated, and when the input magnitude increases asymptotically, the IPC converges to 2.0 because the $z$ component does not respond to input. This is exemplified by the green line orbit of Fig. 1(e). The variations of the degree components are shown in Fig. 4(b). Low-degree $K=1,3$ components have a peak at the edge of chaos. Note that the degree $K=1$ is the MC. However, high-degree components beyond the edge of chaos are higher than ones at the edge of chaos, and there is a trade-off between low- and high-degree components. Moreover, odd-degree $K=1,3, \ldots$ components are dominant in the spintronics reservoir. This suggests that the function from input series to output values is similar to the odd function.

Here, we calculate the MCs, IPCs, and memory functions of only one component in $x, y$, and $z$ of the magnetization. 
(a)
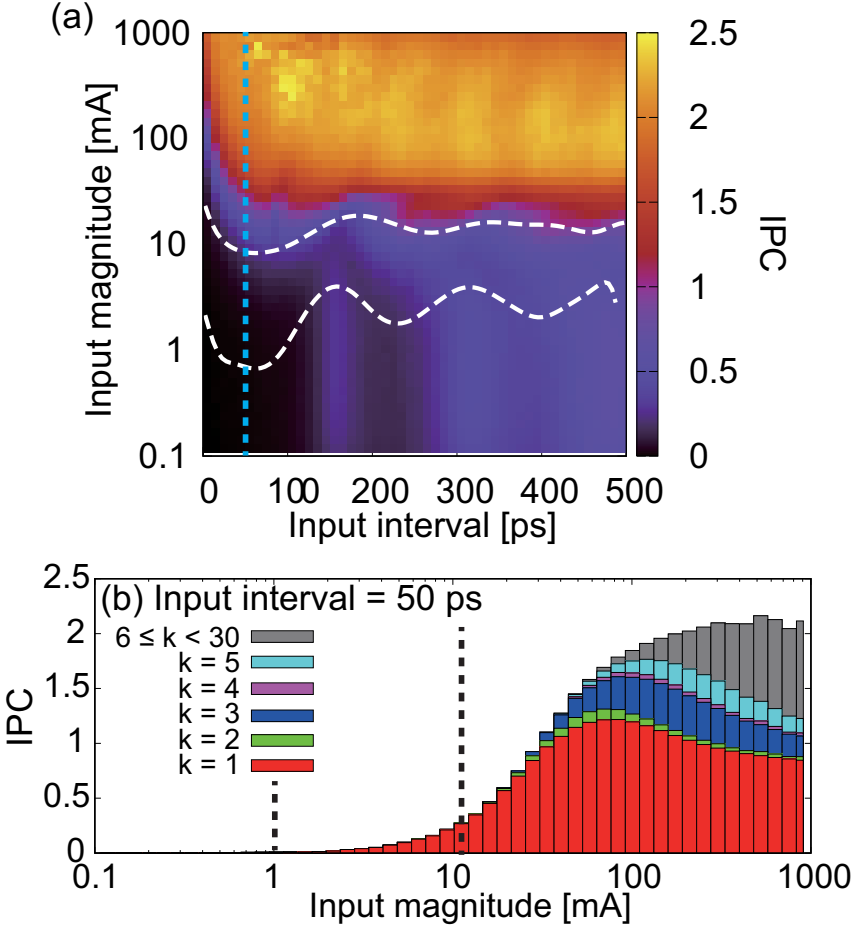

FIG. 4. Analyses of the information processing capacity (IPC). (a) Color map of the IPC. The white lines represent bifurcation points. (b) IPC trade-off of delay with input interval $\tau=50 \mathrm{ps}$. Red, green, blue, purple, light blue, and gray are degree $k=1,2,3,4,5$, and from 6 to 29 , respectively.

Figure 5 shows the $\operatorname{MC}[X, 499]$ and $\operatorname{IPC}[X, 4,29]$ for each of the components. The MCs of the $x$ and $y$ components, which are oscillating variables of the limit cycle, decrease when the input interval is equal to $n T$. In contrast, MCs of the $z$ component, which is almost an orthogonal variable of the limit cycle, are high value in the chaos region when input interval is equal to $n T$. The IPC of the $x$ component converges to 1 as Eq. (9) in the ordered region with large input magnitude; however, IPCs of the $y$ and $z$ components do not converge to 1 . The reason that the IPCs using all the variables investigated in the previous paragraph do not converge is considered to be due to the $y$ and $z$ components. As can be seen from the plots, each variable contains different types of computational capability. Figures 5(g)-5(i) show memory functions with the same parameters as in Fig. 3(f). In these figures, we can clearly find extremely long memory tails and sharp vanishing of memory tails at input intervals $\tau=n T$. Furthermore, memory tails have periodic structure and are composed of strange units, whose lengths are about delay 16 . It may be considered that these phenomena are driven by the original limit cycle dynamics. Further investigations into the details of the behavior of IPC, MC, and memory function are needed.

\section{BENCHMARK TASKS}

Finally, we solve benchmark tasks with the spintronics reservoir and show how these diverse repertoires in magnetization dynamics contribute to the task performance. The tasks
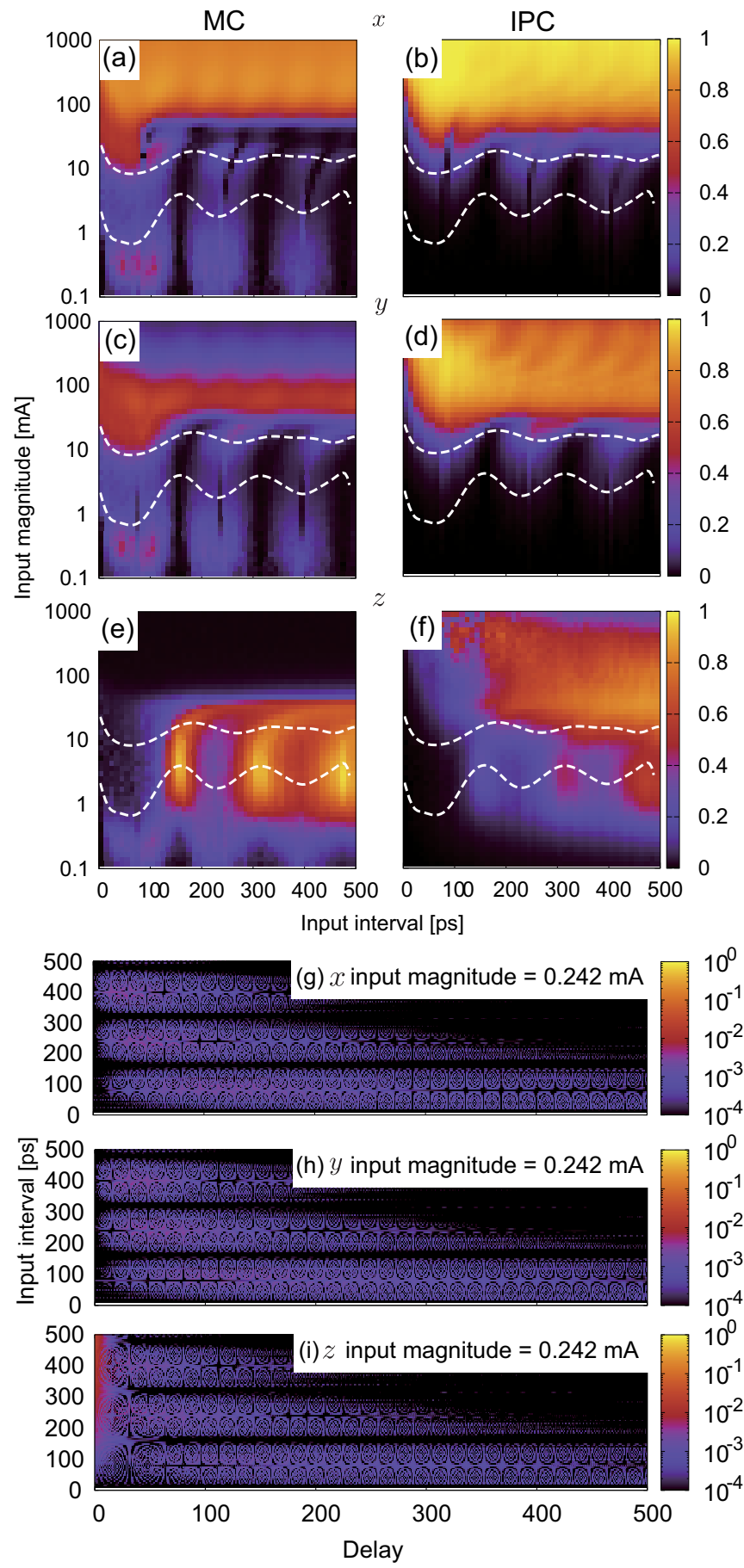

FIG. 5. MCs, IPCs and memory functions of each component in the STO. (a) and (b) are MC and IPC of the $x$ coordinate, respectively. (c) and (d) are MC and IPC of the $y$ coordinate, respectively. (e) and (f) are MC and IPC of the $z$ coordinate, respectively. (g), (h), and (i) are memory functions of the $x, y$, and $z$ coordinates, respectively, with input magnitude $A=0.242 \mathrm{~mA}$. The color represents capacity of the corresponding delay and input interval.

that we focus on are the emulation of a nonlinear dynamical system, called the NARMA2 [37] and a length emulation of PAM [38] (details of these tasks are provided in Appendix E). NARMA2, represented as a difference equation with nonlinear processing of previous inputs, is a model whose emulation 


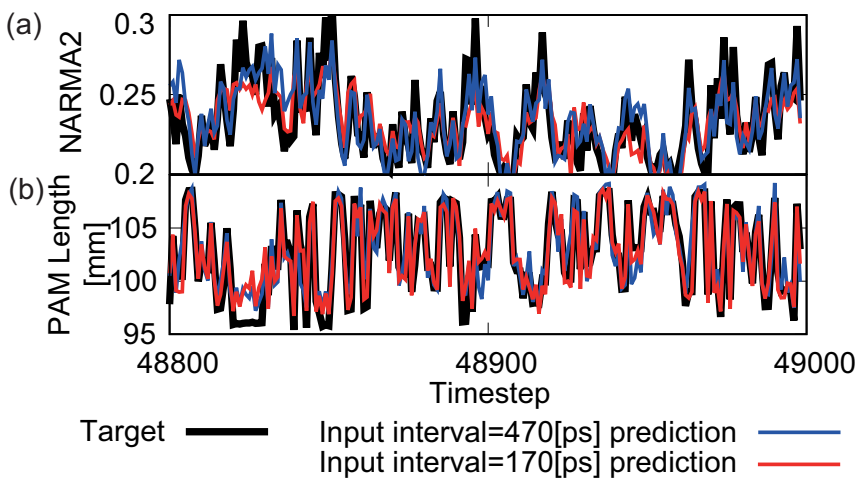

FIG. 6. Typical performances of the spintronics reservoir with some temporal machine-learning tasks. (a) Time series of NARMA2. The black line is the target signal, the blue line is the prediction signal of the spintronics reservoir with input interval $470 \mathrm{ps}$, which is designed for the NARMA2, and the red line is the prediction signal of spintronics reservoir with input interval $170 \mathrm{ps}$ that is designed for the PAM length emulation. (b) Time series of PAM length emulation. The color correspondence is the same as in (a).

capability is widely used as a benchmark in a recurrent neural network $[4,10,11,39]$. The PAM length emulation task is a real-world task for edge computing that emulates the PAM length from an injected pressure value, which is important for soft robotics applications [38]. Short-term memory and nonlinearity are necessary to solve these tasks. By fixing the input interval, 50 types of input magnitudes from 0.1 to 1000 $\mathrm{mA}$ are injected into different spintronics reservoirs driven in parallel, a scheme called spatial multiplexing [11].

Figures 6(a) and 6(b) show the prediction results of the NARMA2 and the PAM length emulations, respectively, where the input signal is injected as an input value for the NARMA2 and pressure in the real experiment for PAM length emulation. We can confirm that each task was solved effectively by the STO, with optimized input settings.

Table I, which shows the normalized mean-square error (NMSE), quantitatively supports the above results. The highest performance input intervals were $470 \mathrm{ps}$ for the NARMA2 and 170 ps for the PAM length emulation. These input intervals were approximately equal to the multiples of the inputless period of the STO, and the chaos region is narrow along the input magnitude within the intervals.

We confirmed the relationship between the NMSE of tasks and their largest capacities by plotting color maps. Since the target time series of each task can be expressed as a function of the previous input sequence, we can also calculate the IPC of each task [49]. Here, target functions of the largest capacity of NARMA2 and PAM tasks are $\mathcal{P}_{1}\left(u_{n-1}\right)$ and $\mathcal{P}_{1}\left(u_{n}\right)$, re-

TABLE I. NMSE for benchmark tasks.

\begin{tabular}{lcc}
\hline \hline & \multicolumn{2}{c}{ Input interval } \\
\cline { 2 - 3 } & $470 \mathrm{ps}$ & $170 \mathrm{ps}$ \\
\hline NARMA2 & 0.2694 & 0.4672 \\
PAM length emulation & 0.1344 & 0.0789 \\
\hline \hline
\end{tabular}

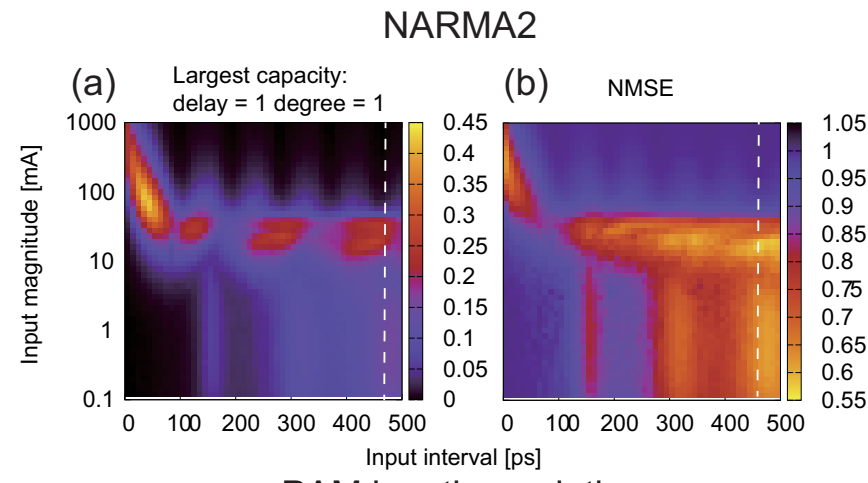

PAM length emulation

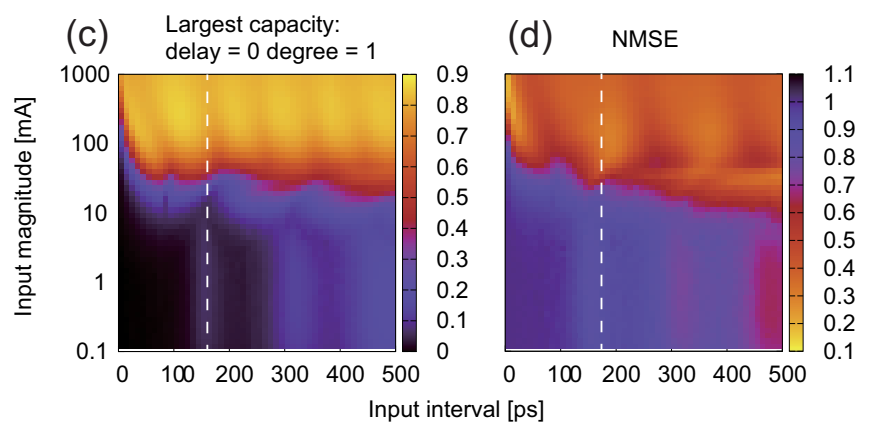

FIG. 7. Color maps of the largest capacity and NMSE of the NARMA2 and PAM length emulations. (a) The largest capacity of the NARMA2 is $C\left[X, \mathcal{P}_{1}\left(u_{n-1}\right)\right]$. (b) NMSE of the NARMA2. (c) The largest capacity of the PAM length emulation is $C\left[X, \mathcal{P}_{1}\left(u_{n}\right)\right]$. (d) NMSE of the PAM length emulation. White lines show the best input interval using the spatial multiplexing method for each task.

spectively. A more detailed analysis of the IPC of each task is given in Appendix E. Figure 7 shows the color maps of the largest capacity and NMSEs. Note that we used the spatial multiplexing method; however, we analyzed a performance of a single spintronics reservoir in Figs. 7(b) and 7(d). In the NARMA2, the reservoir performance improved at the edge of chaos. In the PAM length emulation, the reservoir performance improved in the ESP region after chaos. The NMSEs are improved when the largest capacity is a high value.

\section{CONCLUSION}

In this paper, we revealed two input-driven bifurcations in the STO and confirmed that the information processing capacity property changed drastically through these bifurcations. Even through the internal parameters of the spintronics reservoir are fixed, the STO shows diverse dynamics depending on the input configuration. Therefore we can design the dynamical properties of the spintronics reservoir only by adjusting the input setting after fixing the device configuration. Clarifying the input-driven bifurcations of a device is important for understanding the appropriate input configuration. Additionally, changing the input configuration, such as input magnitude and interval, also changes the energy injected into the system, and we will be able to consider improving the energy efficiency of the devices. 


\section{ACKNOWLEDGMENTS}

The results were partially obtained from a project [Innovative AI Chips and Next-Generation Computing Technology Development/Development of Next-Generation Computing Technologies/Exploration of Neuromorphic Dynamics towards Future Symbiotic Society] commissioned by NEDO. K.N. is supported by JSPS KAKENHI Grant No. JP18H05472.

\section{APPENDIX A: VALUE OF PARAMETERS}

The values of parameters in the LLG equation are derived from an experimental study [23] and a theoretical study [40] and are shown in Table II.

\section{APPENDIX B: LYAPUNOV EXPONENTS}

Here, we introduce how the Lyapunov exponents were measured for the LLG equation. As a premise, we solved the LLG equation using the fourth Runge-Kutta method. The LLG equation holds that $\|\mathbf{m}\|=1$. It has been confirmed that ||m|| does not change with a sufficiently small time increment instead of using, for instance, the symplectic method. The Lyapunov exponents are calculated with the ShimadaNagashima method [50], which is based on an expanding rate of a perturbation vector. Note that the degrees of freedom of the perturbation vector are not 3 as a dimension of $\mathbf{m}$, but 2 because of the constraint $\|\mathbf{m}\|=1$. The calculation of Lyapunov exponents uses a distance between state variables; we chose an angle on the sphere $\|\mathbf{m}\|=1$. See Ref. [32] for details on how to give perturbations on the sphere $\|\mathbf{m}\|=1$ and how to measure distances on the sphere. Figure 2(b) shows the largest Lyapunov exponents in this two-dimensional space.

The LLG equation with time-dependent input signals is a nonautonomous system. Therefore, to calculate the nonconditional Lyapunov exponents, we replaced the state variable with the following equation that adds the time variable to calculate the nonconditional Lyapunov exponents: $\tilde{\mathbf{m}}=(\mathbf{m} t)^{T}$. See Ref. [32] for how to measure the distance of $\tilde{\mathbf{m}}$. Furthermore, our system is a switched (hybrid) dynamical system because of the noncontinuity of the input signal $j_{\text {in. }}$. In a switched dynamical system, the Lyapunov exponents are based on expanding rates in piecewise continuous intervals [51]. This system is constant for a time in piecewise continuous intervals; hence the Lyapunov exponent of the time

TABLE II. Value of parameters.

\begin{tabular}{lcc}
\hline \hline Symbol & Explanation & Value \\
\hline$M$ & Saturation magnetization & $1448.3 \mathrm{emu} / \mathrm{cm}^{3}$ \\
$H_{\mathrm{K}}$ & Interfacial magnetic anisotropy field & $18.616 \mathrm{kOe}$ \\
$H_{\text {appl }}$ & Applied field & $2.0 \mathrm{kOe}$ \\
$V$ & Volume of the free layer & $\pi \times 60^{2} \times 2 \mathrm{~nm}^{3}$ \\
$\eta$ & Spin polarization & 0.537 \\
$\lambda$ & Gyromagnetic ratio & 0.288 \\
$\gamma$ & Spin-transfer torque asymmetry & $1.764 \times 10^{7} \mathrm{rad} /(\mathrm{Oe} \mathrm{s})$ \\
$\alpha$ & Gilbert damping constant & 0.005 \\
$j_{\mathrm{dc}}$ & Constant current & $2.5 \mathrm{~mA}$ \\
\hline \hline
\end{tabular}

direction is always zero and is independent of other Lyapunov exponents. Accordingly, the (nonconditional) Lyapunov exponents are two m-conditional Lyapunov exponents and one time direction Lyapunov exponent that equals zero. The results of calculating the Lyapunov exponents are shown in Fig. 8.

\section{APPENDIX C: COMMON-SIGNAL SYNCHRONIZATION}

We calculate the synchronization index of Eq. (4) in the main as the transient time $R=4.0 \times 10^{5} \mathrm{ps}$ and the evaluation time $S=2.0 \times 10^{3} \mathrm{ps}$. The distance in Eq. (3) is defined as the angle of the sphere, which is the same as the distance in the Lyapunov exponents. The results are the average of five numerical analyses that had different initial points and input signals.

\section{APPENDIX D: MEMORY CAPACITY AND INFORMATION PROCESSING CAPACITY}

\section{The details of the numerical analysis}

Here, we give the details of the numerical analysis of the MC and IPC. We calculated the MC and IPC from the $10^{5}$ data, with the exception of the 1000 washout data. Capacities under the threshold $\varepsilon=2.0 \times 10^{-4}$ are considered to be zero because overestimation caused by finite data calculation cannot be ignored [36]. Orthogonal basis functions with a range $[-1,1]$ follow the Legendre polynomials:

$$
\mathcal{P}_{n}(u)=\sqrt{\frac{2 n+1}{2}} \frac{(-1)^{n}}{2^{n} !} \frac{d^{n}}{d u^{n}}\left(1-u^{2}\right)^{n} \quad(n=0,1, \ldots) .
$$

\section{Asymptotic value of memory capacity}

In Figs. 3(b) and 3(c), the MC converges to a constant when the input magnitude is sufficiently large. We estimate the convergence value from the magnetization dynamics. When the input magnitude is sufficiently large, the magnetization switching occurs, and the magnetization converges to $\mathbf{m}=$ $(-1,0,0)$ or $\mathbf{m}=(1,0,0)$, depending on the sign of the input signal value. Therefore capacities with nonzero delay are always zero, and the capacities with zero delay have the following value:

$$
\begin{aligned}
C\left[X, u_{n}\right] & =\frac{\left\langle u_{n} \mathbf{x}_{n}\right\rangle^{T}\left\langle\mathbf{x}_{n} \mathbf{x}_{n}^{T}\right\rangle^{-1}\left\langle u_{n} \mathbf{x}_{n}\right\rangle}{\left\langle y_{n}^{2}\right\rangle} \\
& =\frac{\left\langle u_{n}\left[-\operatorname{sgn}\left(u_{n}\right)\right]\right\rangle^{2}}{\left\langle u_{n}^{2}\right\rangle\left\langle\left[-\operatorname{sgn}\left(u_{n}\right)\right]^{2}\right\rangle} \\
& =\frac{1^{2}}{\frac{2}{3} \times 2}=0.75,
\end{aligned}
$$

where we use the fact that the component depending on $u_{n}$ is only $m_{x}$ and its value $-\operatorname{sgn}\left(u_{n}\right)$ to calculate from the first line to the second. Note that the theoretical value of 0.75 is slightly smaller than the experimental value in Fig. 3 because when the random value of input signal is nearly zero, the dynamics do not converge to the fixed point but rather to the limit cycle. 

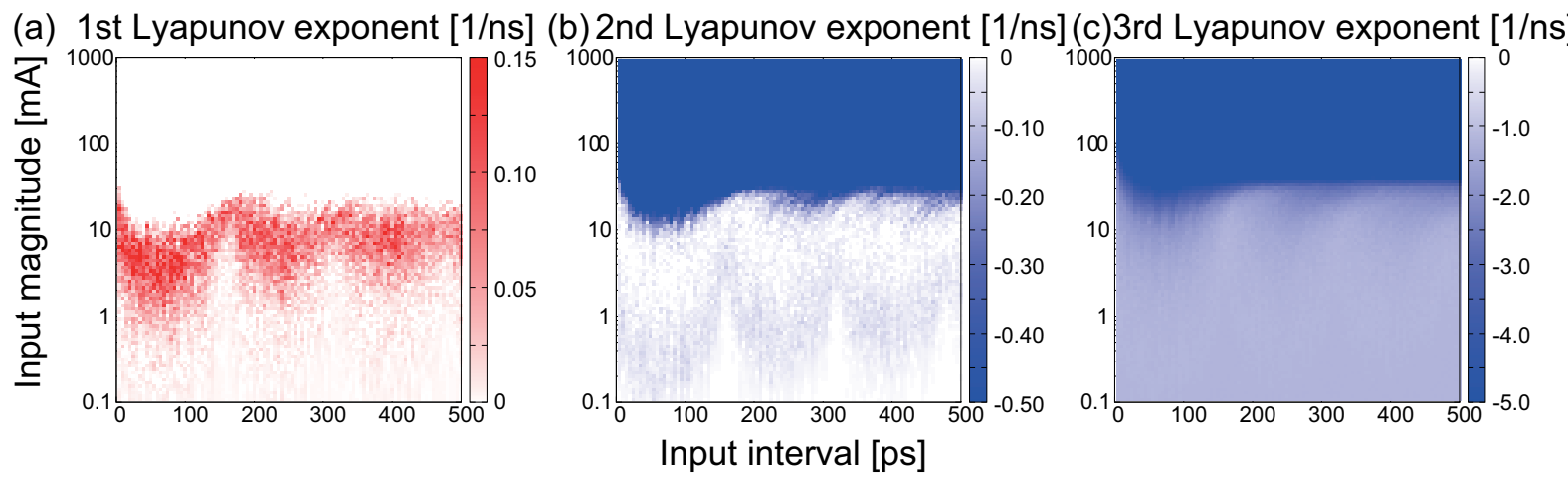

FIG. 8. Color maps of the (a) first Lyapunov exponent, (b) second Lyapunov exponent, and (c) third Lyapunov exponent.

\section{APPENDIX E: BENCHMARK TASKS}

\section{NARMA2}

In this section, we introduce the NARMA2 task. The NARMA2 is a virtual benchmark that tests memory and nonlinear processing capacity of a recurrent neural network [37]. It is represented as the following second-order difference equation:

$$
\begin{gathered}
y_{k+1}=\alpha y_{k}+\beta y_{k} y_{k-1}+\gamma z_{k}^{3}+\delta, \\
z_{k}=\mu+\sigma u_{k},
\end{gathered}
$$

where $\left\{y_{k}\right\}_{k=1, \ldots}$ is the target signal, $\left\{u_{k}\right\}_{k=0, \ldots}$ is the input signal, and $(\alpha, \beta, \gamma, \delta, \mu, \sigma)$ are parameters of NARMA2. We used the parameters $(\alpha, \beta, \gamma, \delta, \mu, \sigma)=$ $(0.4,0.4,0.6,0.1,0.25,0.25)[23,37,52]$.

\section{PAM length emulation}

Here, we introduce the PAM length emulation task. The McKibben PAM is frequently used as a practical soft robot device because of its advantages of being ultralightweight and powerful. The dynamics of PAM are very complex for high dimensionality and nonlinearity; hence the length of PAM is usually measured by an infrared sensor [38]. However, there is a problem because the infrared sensor, as a rigid device, loses the softness of the PAM. The physics model-based method is also not practical because it requires estimating many parameters [53]. However, an echo state network can solve this task with the same accuracy as an infrared sensor [38]. Since this task aims to replace the sensor, it can be said to be an edge-computing task suitable for reservoir computing. The input signal of this task is a pressure value that is injected in the PAM, and the target signal is the length of the PAM at the time of switching to the next input signal. We obtained data from an experiment involving an actual PAM device. Here,

TABLE III. NARMA2's largest capacities.

\begin{tabular}{lc}
\hline \hline Target function & Capacity value \\
\hline $\mathcal{P}_{1}\left(u_{n-1}\right)$ & 0.5847 \\
$\mathcal{P}_{1}\left(u_{n-2}\right)$ & 0.1453 \\
$\mathcal{P}_{2}\left(u_{n-1}\right)$ & 0.1109 \\
$\mathcal{P}_{1}\left(u_{n-3}\right)$ & 0.0697 \\
$\mathcal{P}_{1}\left(u_{n-4}\right)$ & 0.0292 \\
\hline \hline
\end{tabular}

the input signal is linearly transformed to the $[0.0,0.5] \mathrm{MPa}$ range. The input signal switches every $0.3 \mathrm{~s}$. The load that is added to the PAM is 100 N. See Ref. [38] for further details of the experiment. The training and evaluation data sets used in this experiment are provided as Supplemental Material [54].

\section{Details of the numerical analysis}

We injected the same input signal into spintronics reservoirs in both tasks and predicted target signals from the reservoir output signals. We adopted the spatial multiplexing method [11] to improve performance. In this case, we injected the same input signal to 50 different spintronics reservoirs, and output signals of the spintronics reservoirs were used for the prediction in parallel. The 49998-input data set of uniform random variables with an interval of $[-1,1]$ was split into 998 for the washout data, 40000 for the training data, and 9000 for the evaluation data. We calculated the normalized mean-square error as follows:

$$
\mathrm{NMSE}=\frac{1}{9000} \frac{\sum_{k=40999}^{49998}\left(y_{k}-\hat{y}_{k}\right)^{2}}{\sum_{k=40999}^{49998} y_{k}^{2}},
$$

where $y_{k}$ is the target signal and $\hat{y}_{k}$ is the prediction value of spintronics reservoirs.

\section{Properties of tasks}

In both tasks, we calculated the IPCs of the target signal for the input signal. If the ESP was held, the IPC equaled 1.0, and we reconstructed the function from the input signal to the target signal [49]. IPC $[X, D, K]$ with the maximum delay $D=$ 6 and maximum degree $K=5$ had the following values:

$$
\begin{gathered}
\operatorname{IPC}_{\text {NARMA2 }}[X, 6,5]=1.009, \\
\operatorname{IPC}_{\text {PAM }}[X, 6,5]=0.964 .
\end{gathered}
$$

TABLE IV. The PAM length emulation's largest capacities.

\begin{tabular}{lc}
\hline \hline Target function & Capacity value \\
\hline $\mathcal{P}_{1}\left(u_{n}\right)$ & 0.6278 \\
$\mathcal{P}_{1}\left(u_{n-1}\right)$ & 0.2583 \\
$\mathcal{P}_{1}\left(u_{n-2}\right)$ & 0.0389 \\
$\mathcal{P}_{2}\left(u_{n}\right) \mathcal{P}_{1}\left(u_{n-1}\right)$ & 0.091 \\
$\mathcal{P}_{2}\left(u_{n-1}\right) \mathcal{P}_{1}\left(u_{n-2}\right)$ & 0.085 \\
\hline \hline
\end{tabular}


Both IPCs are sufficiently close to 1.0; hence the maximum delay and maximum degree are considered appropriate. The reason that $\mathrm{IPC}_{\mathrm{NARMA} 2}$ slightly exceeds 1.0 is because of an accumulation of overestimation, and the reason that $\mathrm{IPC}_{\mathrm{PAM}}$ is below 1.0 may be due to the loss of ESP by noise. The top five capacities of both tasks are shown in Tables III and IV, where $\mathcal{P}_{k}\left(u_{n-d}\right)$ is the Legendre polynomial with the degree $k$ and the argument $u_{n-d}$.
[1] W. Maass, T. Natschläger, and H. Markram, Real-time computing without stable states: A new framework for neural computation based on perturbations, Neural Comput. 14, 2531 (2002).

[2] H. Jaeger and H. Haas, Harnessing nonlinearity: Predicting chaotic systems and saving energy in wireless communication, Science 304, 78 (2004).

[3] K. Nakajima, Physical reservoir computing - an introductory perspective, Jpn. J. Appl. Phys. 59, 060501 (2020).

[4] K. Nakajima, H. Hauser, T. Li, and R. Pfeifer, Information processing via physical soft body, Sci. Rep. 5, 10487 (2015).

[5] K. Nakajima, H. Hauser, T. Li, and R. Pfeifer, Exploiting the dynamics of soft materials for machine learning, Soft Robotics 5, 339 (2018).

[6] L. Larger, M. C. Soriano, D. Brunner, L. Appeltant, J. M. Gutiérrez, L. Pesquera, C. R. Mirasso, and I. Fischer, Photonic information processing beyond turing: An optoelectronic implementation of reservoir computing, Opt. Express 20, 3241 (2012).

[7] D. Brunner, M. C. Soriano, C. R. Mirasso, and I. Fischer, Parallel photonic information processing at gigabyte per second data rates using transient states, Nat. Commun. 4, 1364 (2013).

[8] K. Vandoorne, P. Mechet, T. Van Vaerenbergh, M. Fiers, G. Morthier, D. Verstraeten, B. Schrauwen, J. Dambre, and P. Bienstman, Experimental demonstration of reservoir computing on a silicon photonics chip, Nat. Commun. 5, 3541 (2014).

[9] L. Larger, A. Baylón-Fuentes, R. Martinenghi, V. S. Udaltsov, Y. K. Chembo, and M. Jacquot, High-speed photonic reservoir computing using a time-delay-based architecture: Million words per second classification, Phys. Rev. X 7, 011015 (2017).

[10] K. Fujii and K. Nakajima, Harnessing Disordered-Ensemble Quantum Dynamics for Machine Learning, Phys. Rev. Appl. 8, 024030 (2017).

[11] K. Nakajima, K. Fujii, M. Negoro, K. Mitarai, and M. Kitagawa, Boosting Computational Power through Spatial Multiplexing in Quantum Reservoir Computing, Phys. Rev. Appl. 11, 034021 (2019).

[12] S. Ghosh, T. Paterek, and T. C. H. Liew, Quantum Neuromorphic Platform for Quantum State Preparation, Phys. Rev. Lett. 123, 260404 (2019)

[13] J. Torrejon, M. Riou, F. A. Araujo, S. Tsunegi, G. Khalsa, D. Querlioz, P. Bortolotti, V. Cros, K. Yakushiji, A. Fukushima, H. Kubota, S. Yuasa, M. D. Stiles, and J. Grollier, Neuromorphic computing with nanoscale spintronic oscillators, Nature (London) 547, 428 (2017).

[14] T. Furuta, K. Fujii, K. Nakajima, S. Tsunegi, H. Kubota, Y. Suzuki, and S. Miwa, Macromagnetic Simulation for Reservoir Computing Utilizing Spin Dynamics in Magnetic Tunnel Junctions, Phys. Rev. Appl. 10, 034063 (2018).

[15] S. Tsunegi, T. Taniguchi, S. Miwa, K. Nakajima, K. Yakushiji, A. Fukushima, S. Yuasa, and H. Kubota, Evaluation of memory capacity of spin torque oscillator for recurrent neural networks, Jpn. J. Appl. Phys. 57, 120307 (2018).
[16] S. Tsunegi, T. Taniguchi, K. Nakajima, S. Miwa, K. Yakushiji, A. Fukushima, S. Yuasa, and H. Kubota, Physical reservoir computing based on spin torque oscillator with forced synchronization, Appl. Phys. Lett. 114, 164101 (2019).

[17] W. Jiang, L. Chen, K. Zhou, L. Li, Q. Fu, Y. Du, and R. Liu, Physical reservoir computing using magnetic skyrmion memristor and spin torque nano-oscillator, Appl. Phys. Lett. 115, 192403 (2019).

[18] D. Marković, A. Mizrahi, D. Querlioz, and J. Grollier, Physics for neuromorphic computing, Nat. Rev. Phys. 2, 499 (2020).

[19] T. Yamaguchi, N. Akashi, S. Tsunegi, H. Kubota, K. Nakajima, and T. Taniguchi, Periodic structure of memory function in spintronics reservoir with feedback current, Phys. Rev. Research 2, 023389 (2020).

[20] D. Kobayashi, K. Hirose, T. Makino, S. Onoda, T. Ohshima, S. Ikeda, H. Sato, E. C. I. Enobio, T. Endoh, and H. Ohno, Soft errors in 10-nm-scale magnetic tunnel junctions exposed to high-energy heavy-ion radiation, Jpn. J. Appl. Phys. 56, 0802B4 (2017).

[21] J. A. Katine, F. J. Albert, R. A. Buhrman, E. B. Myers, and D. C. Ralph, Current-Driven Magnetization Reversal and SpinWave Excitations in $\mathrm{Co} / \mathrm{Cu} / \mathrm{Co}$ Pillars, Phys. Rev. Lett. 84, 3149 (2000).

[22] S. I. Kiselev, J. Sankey, I. Krivorotov, N. Emley, R. Schoelkopf, R. Buhrman, and D. Ralph, Microwave oscillations of a nanomagnet driven by a spin-polarized current, Nature (London) 425, 380 (2003).

[23] H. Kubota, K. Yakushiji, A. Fukushima, S. Tamaru, M. Konoto, T. Nozaki, S. Ishibashi, T. Saruya, S. Yuasa, T. Taniguchi, H. Arai, and H. Imamura, Spin-torque oscillator based on magnetic tunnel junction with a perpendicularly magnetized free layer and in-plane magnetized polarizer, Appl. Phys. Express 6, 103003 (2013).

[24] J. C. Slonczewski, Current-driven excitation of magnetic multilayers, J. Magn. Magn. Mater. 159, L1 (1996).

[25] L. Berger, Emission of spin waves by a magnetic multilayer traversed by a current, Phys. Rev. B 54, 9353 (1996).

[26] S. Kaka, M. R. Pufall, W. H. Rippard, T. J. Silva, S. E. Russek, and J. A. Katine, Mutual phase-locking of microwave spin torque nano-oscillators, Nature (London) 437, 389 (2005).

[27] F. Mancoff, N. Rizzo, B. Engel, and S. Tehrani, Phase-locking in double-point-contact spin-transfer devices, Nature (London) 437, 393 (2005).

[28] A. Slavin and V. Tiberkevich, Nonlinear auto-oscillator theory of microwave generation by spin-polarized current, IEEE Trans. Magn. 45, 1875 (2009).

[29] Z. Yang, S. Zhang, and Y. C. Li, Chaotic Dynamics of SpinValve Oscillators, Phys. Rev. Lett. 99, 134101 (2007).

[30] K. Nakada, S. Yakata, and T. Kimura, Noise-induced synchronization in spin torque nano oscillators, J. Appl. Phys. 111, 07C920 (2012).

[31] J. Williame, A. Difini Accioly, D. Rontani, M. Sciamanna, and J.-V. Kim, Chaotic dynamics in a macrospin spin-torque 
nano-oscillator with delayed feedback, Appl. Phys. Lett. 114, 232405 (2019).

[32] T. Yamaguchi, N. Akashi, K. Nakajima, S. Tsunegi, H. Kubota, and T. Taniguchi, Synchronization and chaos in a spin-torque oscillator with a perpendicularly magnetized free layer, Phys. Rev. B 100, 224422 (2019).

[33] T. Taniguchi, N. Akashi, H. Notsu, M. Kimura, H. Tsukahara, and K. Nakajima, Chaos in nanomagnet via feedback current, Phys. Rev. B 100, 174425 (2019).

[34] I. B. Yildiz, H. Jaeger, and S. J. Kiebel, Re-visiting the echo state property, Neural Networks 35, 1 (2012).

[35] H. Jaeger, Short Term Memory in Echo State Networks (GMD-Forschungszentrum Informationstechnik, Sankt Augustin, 2001), Vol. 5.

[36] J. Dambre, D. Verstraeten, B. Schrauwen, and S. Massar, Information processing capacity of dynamical systems, Sci. Rep. 2 , 514 (2012).

[37] A. F. Atiya and A. G. Parlos, New results on recurrent network training: Unifying the algorithms and accelerating convergence, IEEE Trans. Neural Networks 11, 697 (2000).

[38] R. Sakurai, M. Nishida, H. Sakurai, Y. Wakao, N. Akashi, Y. Kuniyoshi, Y. Minami, and K. Nakajima, Emulating a sensor using soft material dynamics: A reservoir computing approach to pneumatic artificial muscle, in 2020 3rd IEEE International Conference on Soft Robotics (RoboSoft) (IEEE, Piscataway, NJ, 2020), pp. 710-717.

[39] L. Appeltant, M. C. Soriano, G. Van der Sande, J. Danckaert, S. Massar, J. Dambre, B. Schrauwen, C. R. Mirasso, and I. Fischer, Information processing using a single dynamical node as complex system, Nat. Commun. 2, 468 (2011).

[40] T. Taniguchi, T. Ito, S. Tsunegi, H. Kubota, and Y. Utsumi, Relaxation time and critical slowing down of a spin-torque oscillator, Phys. Rev. B 96, 024406 (2017).

[41] J. P. Crutchfield, J. D. Farmer, and B. A. Huberman, Fluctuations and simple chaotic dynamics, Phys. Rep. 92, 45 (1982).

[42] R. Toral, C. R. Mirasso, E. Hernández-Garcia, and O. Piro, Analytical and numerical studies of noise-induced synchronization of chaotic systems, Chaos 11, 665 (2001).

[43] D. Snyder, A. Goudarzi, and C. Teuscher, Computational capabilities of random automata networks for reservoir computing, Phys. Rev. E 87, 042808 (2013).

[44] Z. Lu, B. R. Hunt, and E. Ott, Attractor reconstruction by machine learning, Chaos 28, 061104 (2018).

[45] L. M. Pecora and T. L. Carroll, Synchronization in Chaotic Systems, Phys. Rev. Lett. 64, 821 (1990).

[46] N. Bertschinger and T. Natschläger, Real-time computation at the edge of chaos in recurrent neural networks, Neural Comput. 16, 1413 (2004).

[47] J. Schuecker, S. Goedeke, and M. Helias, Optimal Sequence Memory in Driven Random Networks, Phys. Rev. X 8, 041029 (2018).

[48] T. Haruna and K. Nakajima, Optimal short-term memory before the edge of chaos in driven random recurrent networks, Phys. Rev. E 100, 062312 (2019).

[49] T. Kubota, K. Nakajima, and H. Takahashi, Dynamical anatomy of NARMA10 benchmark task, arXiv:1906.04608.

[50] I. Shimada and T. Nagashima, A numerical approach to ergodic problem of dissipative dynamical systems, Prog. Theor. Phys. 61, 1605 (1979).

[51] M.-F. Danca, Synchronization of switch dynamical systems, Int. J. Bifurcation Chaos Appl. Sci. Eng. 12, 1813 (2002).

[52] K. Nakajima, H. Hauser, R. Kang, E. Guglielmino, D. G. Caldwell, and R. Pfeifer, A soft body as a reservoir: Case studies in a dynamic model of octopus-inspired soft robotic arm, Front. Comput. Neurosci. 7, 91 (2013).

[53] H. Schulte, Jr., The characteristics of the McKibben artificial muscle, in The Application of External Power in Prosthetics and Orthotics (National Academy of Sciences-National Research Council, Washington, DC, 1961), Appendix H, p. 94.

[54] See Supplemental Material at http://link.aps.org/supplemental/ 10.1103/PhysRevResearch.2.043303 for all the time series data required for the length emulation task of the pneumatic artificial muscle. 